\section{El Modelo Analítico Tridimensional obtenido por fotogrametría. Descomposición, manipulación y aplicaciones en el campo de la restauración arquitectónica*}

LeANdRo Cámara, Pablo Latorre

Arquitectos, Madrid.

\begin{abstract}
Resumen
Los edificios históricos presentan una forma y una geometría deformada y erosionada que aumenta la complejidad tridimensional de su estructura. La fotogrametría analítica y su registro en programas de CAD permite dibujar modelos analíticos 3D completos de los edificios. En éstos se representan todas las superficies visibles en un solo dibujo y en un sistema de coordenadas único, lo que permite situar cada línea en su posición real en el espacio. Estos modelos de líneas son transparentes, lo que impide ocultar las líneas que su sitúan en un segundo plano, y ocupan gran cantidad de memoria, lo que dificulta su manipulación informática. Para resolver ese problema es necesario descomponer el modelo 3D por archivos y capas de dibujo. Cada archivo de dibujo incluye las líneas restituidas de un elemento constructivo y se identifica por la tipología del elemento y su posición en el espacio del edificio. Las líneas se separan en capas según su orientación geográfica y su tipología (arista, contorno, fisura, etc.). La gestión del conjunto de archivos que componen el modelo $3 \mathrm{D}$ se realiza directamente por designación o gráficamente mediante un plano guía simplificado en el que cada línea es una llamada a un archivo. El modelo 3D analítico en una maqueta informática del monumento que nos permite visualizaciones y análisis métricos, geométricos, constructivos y estructurales imposibles sin esta herramienta. Al conectar

informáticamente cada una de las entidades dibujadas con un sistema de bases de datos externo de los estudios del monumento se establecerá lo que conocemos como «Monument Information System», MIS
\end{abstract}

Palabras clave: arquitectura histórica, fotogrametría analítica, CAD, modelo 3D, sistemas de representación, documentación.

\begin{abstract}
Historic buildings present a deformed and eroded shape and geometry which increases the three-dimensional complexity of their structure. Analytical photogrammetry and its register in CAD programmes make it possible to draw complete analytical 3D models of the buildings. These represent all of the visible surfaces in a single drawing in a unique coordinates system, which makes it possible to place each line in its real spatial position. These models of lines are transparent, which prevents background lines from being hidden, and use a great deal of memory, which hinders their IT use. To resolve this problem it is necessary to break down the 3D model into files and drawing layers. Each drawing file includes the restored lines of a constructive element and is identified by the typology of the element and its position in the space of the building. The lines are separated into layers according to their geographic orientation and typology (arris, contour, fissure, etc.) The administration of the set of files making up the 3D model is carried out directly by designation or graphically via a simplified guide map in which each line pertains to a file. The 3D analytic model is an IT model of the monument that permits us metric, geometric constructive and structural visualizations and analyses which would be impossible without this tool. By connecting through information technology each of these drawn entities with an external system of databases of studies of the monument, it will be possible to establish what we know as a «Monument Information System» or MIS.
\end{abstract}

Key Words: historic architecture, analytical photogrammetry, CAD, 3D model, representation systems, documentation.
"... La escultura actúa en tres dimensiones, pero el hombre permanece al exterior, separado, mirándolas desde fuera. La arquitectura, por el contrario, es como una gran escultura excavada, en cuyo interior el hombre penetra y camina"

"..., la realidad del objeto no se agota en las tres dimensiones de la perspectiva; para representarla integralmente tendría que hacerse un sinfin de perspectivas desde los infinitos puntos de vista. Hay, por tanto, otro elemento, además de las tres dimensiones tradicionales, y es, precisamente el desplazamiento sucesivo de ángulo visual. Asi fue bautizado el tiempo como "cuarta dimensión»..."

"...el método de representación de los edificios que encontramos aplicado en la mayoría de las historias del arte y de la arquitectura se sirve de: a) plantas; b) alzados y secciones; c) fotografias. Hemos afirmado que estos medios, considerados aisladamente o en su conjunto, son insuficientes para representar completamente el espacio arquitectónico; sin embargo, es útil profundizar en este problema, ya que — si, hasta ahora no tenemos mejores sistemas de representación - nuestro trabajo es estudiar las técnicas que poseemos y hacerlas más eficaces». De "Saper vedere l'architettura", (Bruno Zevi, 1949, 1991).

\section{LA CONDICIÓN TRIDIMENSIONAL DE LA ARQUITECTURA Y SU REPRESENTACIÓN GRÁFICA}

De todos los objetos manufacturados por el hombre, la arquitectura es el que posee unas características espaciales y tridimensionales más importantes; ya que está concebido para ser utilizado introduciéndose en su interior hueco. Esta condición envolvente de la arquitectura permite al hombre aislarse del espacio exterior abierto e introducirse - como en una cueva - en un espacio cerrado, modelado y construido por él, con las dimensiones, forma, colores y texturas adecuadas para desarrollar una actividad concreta. La arquitectura es un gran volumen construido, con una forma exterior determinada por los espacios que se configuran en su interior; lo que le otorga su condición tridimensional. Estas características y sus dimensiones impiden que podamos comprender una arquitectura observándola desde un único punto de vista y que tengamos que recorrerla, rodeando su perímetro exterior y accediendo en el interior a cada uno de sus espacios, para visualizar la totalidad de su estructura.

Esta complejidad espacial del objeto arquitectónico nos obligará también en los procesos de diseño y proyecto a descomponer su configuración y a utilizar planos y maquetas para representar cada uno de sus elementos, facilitando

\footnotetext{
* El presente artículo es traducción del publicado en inglés en el XVIII Internacinal Simposium of CIPA (The ICOMOS/ISPRS Committe for Documentation of Cultural Heritage), celebrado en Potsdam (Alemania) en el año 2001, donde le fue concedido el Premio al Mejor Poster.
} 


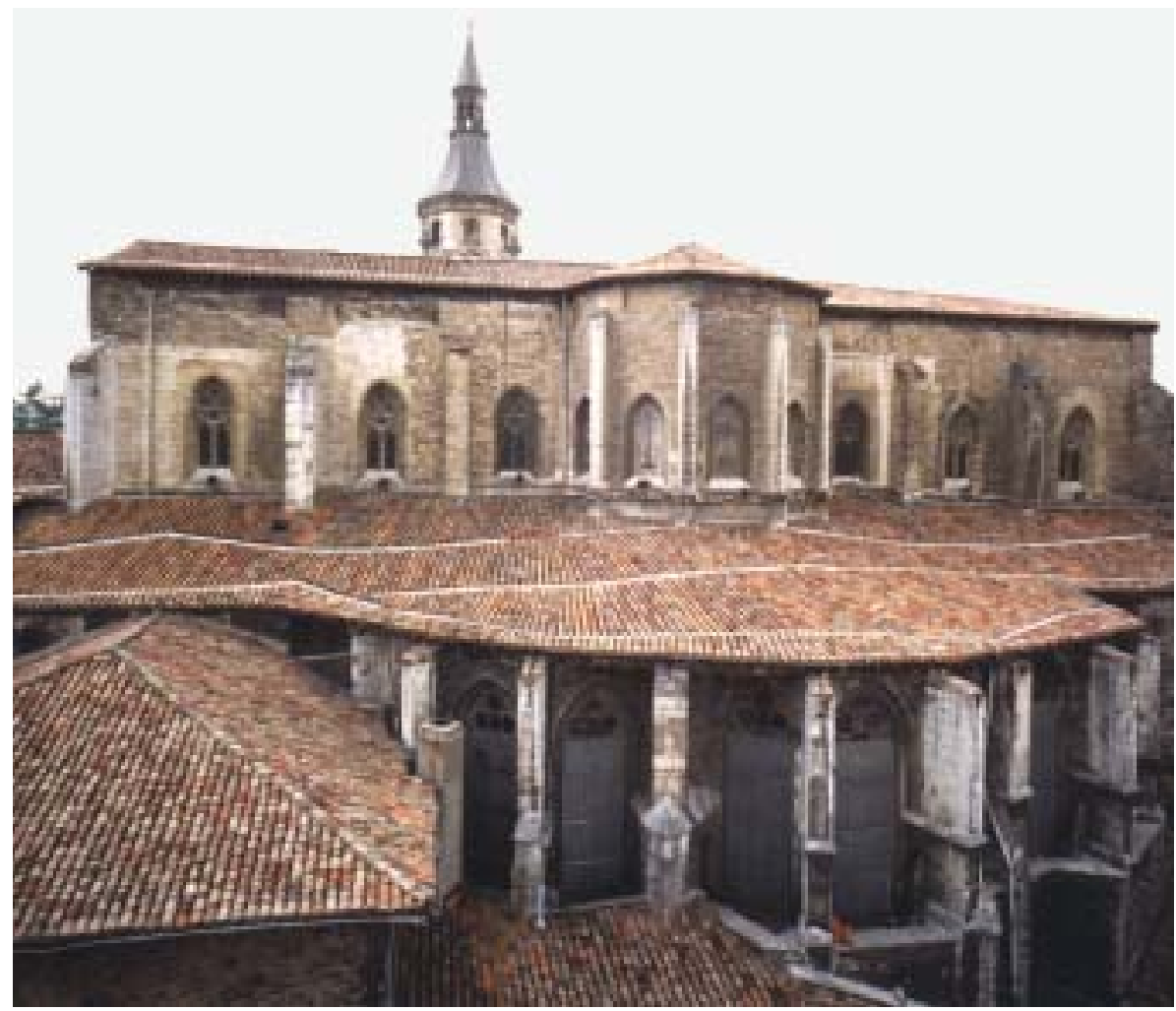

así su comprensión. Para recoger toda la complejidad espacial de una estructura arquitectónica la proyectamos sobre una sucesión de planos horizontales y verticales que seccionan su volumen en paralelo a los planos más significativos de su estructura. Este sistema, habitual para la representación de la arquitectura mediante plantas, alzados y secciones, niega su visión tridimensional provocando que los elementos que no sean paralelos a estos planos de sección y además estén inclinados, tengan un espesor variable o curvatura, sean difícilmente comprensibles en su representación. Las perspectivas nos permiten una representación más aproximada a como percibimos la tridimensionalidad del objeto arquitectónico en la realidad; sin embargo, no permiten una representación métrica y a escala precisa. El levantamiento de un edificio debe combinar la utilización de ambos sistemas de representación para conseguir que el conocimiento del objeto sea él mas adecuado a su condición tridimensional.

\section{FORMA TEÓRICA Y FORMA REAL DE LA ARQUITECTURA HISTÓRICA}

La arquitectura histórica evoluciona siguiendo procesos de transformación complejos, consecuencia de los dilatados periodos de tiempo en los que se han producido y que, inevitablemente, acaban provocando grandes diferencias entre la forma teórica con la que se imaginó y construyó un edi- ficio y la forma real con la que éste llega hasta nosotros. Los defectos y cambios del proyecto en el momento de la construcción, las lesiones y deformaciones producidas por su puesta en carga o por una deficiente concepción de su arquitectura, las lesiones y erosiones debidas a acciones externas naturales o humanas o las transformaciones de su estructura provocadas por los cambios de uso van modificando, a lo largo del tiempo, su forma y su estructura original.

Las diferencias entre la forma teórica y la forma real de un edificio tiene una relación directa con la transformación que ha sufrido en el tiempo, y de la comparación entre ambas podrán deducirse aspectos de su historia, su construcción, sus patologías, etc. Por ejemplo, todos entendemos que un edificio se construye normalmente con una geometría ortogonal, por tanto, la geometría deformada que presenta nos revela los movimientos del edificio acumulados a lo largo de su historia. Sin embargo, la definición de la forma teórica de un edifico histórico - siempre que no exista ningún documento o prueba de como fue construido- no es un dato conocido ni objetivo, y su deducción debe realizarse a partir de la estructura conservada y de los estudios realizados sobre sus características, su historia, sus patologías, etc.

De este modo, el estudio y restauración de un edificio histórico debe iniciarse con el estudio exhaustivo de su estructura, tal y como ha llegado hasta nosotros, con todas 
sus características geométricas, físicas, constructivas, mecánicas o funcionales y con la investigación del proceso histórico que la ha generado a partir de una estructura previa cuya configuración desconocemos. Lógicamente, el punto de partida de este proceso es la realización del levantamiento del edificio histórico conservado, registrando de un modo objetivo y sistemático todas las aristas existentes, el contorno de los materiales y de todos los elementos superficiales como las erosiones o las fisuras, etc. En este punto, la fotogrametría se convierte en una herramienta fundamental de este proceso, ya que permite dibujar con precisión una geometría deformada y erosionada que es difícil de apreciar a simple vista y que, normalmente, no se sujeta a una geometría ortogonal. El registro fotogramétrico impide también, por su sistema de registro indirecto, simplificaciones y falsificaciones de la forma y de las características reales del objeto representado. Desde esta perspectiva, el levantamiento se convierte en la primera forma de conocimiento de un monumento y en la herramienta más importante de todo el proceso de su investigación y restauración.

Es de sobra conocida la importancia que se otorga a todo el abanico de estudios previos sobre un edificio histórico que nos permiten definir sus características (formales, constructivas y funcionales), su proceso histórico y sus problemas y patologías. La información acopiada en este proceso, referida a los materiales, patologías, técnicas constructivas, movimientos, historia, etc., tiene un reflejo gráfico en el levantamiento en forma de planos temáticos. El conjunto de estos planos formará un atlas del monumento similar a los referidos al territorio. La sistematización e informatización de toda esta información referida al edificio en forma de bases de datos y su puesta en relación con el levantamiento mediante un sistema de doble dirección que enlaza los registros gráficos con los registros de información textual y numérica, acompañado de un sistema de gestión, conformará lo que hemos designado como el Sistema de Información Monumental MIS (Monument Information System), en paralelo con la designación establecida para los Sistemas de Información Geográfica GIS (Geografical Information System).

\section{EL MODELO ANALÍTICO 3D OBTENIDO POR FOTOGRAMETRÍA}

La teoría matemática de la fotogrametría ha sido desde su formulación de carácter tridimensional; sin embargo, el registro de la restitución fotogramétrica ha tenido en la práctica un carácter gráfico (analógico) y por tanto bidimensional. Esta limitación del sistema obligaba a que este registro gráfico se realizase siguiendo el sistema de proyección diédrico que era el único —entre los sistemas de representa- ción arquitectónica - que permitía el registro métrico y a escala de las coordenadas (X, Y) del corte que se proyectaba. Durante la restitución únicamente se podía añadir la profundidad de puntos determinados mediante la incorporación escrita del valor de la coordenada $Z$, y sólo en ocasiones se intentó la representación tridimensional de objetos escultóricos o de la geometría de las bóvedas siguiendo el sistema de curvas de nivel utilizado en las cartografías de territorio.

Como es conocido, la revolución informática transformó en analíticos los sistemas de cálculo fotogramétrico aprovechando toda la capacidad tridimensional de su ecuación. Paralelamente, el desarrollo de los programas de dibujo asistido por ordenador (CAD) ha permitido registrar en tiempo real el resultado tridimensional de la restitución y obtener múltiples proyecciones planas —en la pantalla o sobre papel- del modelo restituido. El registro en los sistemas de CAD permite también, mediante un apoyo topográfico adecuado, establecer un sistema de coordenadas único y situar — en un sólo dibujo- cada elemento restituido en su posición real en el espacio. De este modo, podremos ir "construyendo" la maqueta virtual del edificio a medida que vayamos insertando los resultados de la restitución hasta completar la totalidad de las superficies que componen su estructura. El resultado es en realidad un modelo matemático; una suma muy elevada de coordenadas en el espacio unidas por líneas que reproducen la posición exacta de los puntos y las líneas restituidas de la realidad y que contiene toda la información métrica del edificio. Esta maqueta es la que visualiza el programa de $\mathrm{CAD}$ en la pantalla, una vez que se han definido los elementos de la proyección (sistema de representación, vértice y plano de la proyección). De la combinación de estos valores, resultan múltiples visualizaciones del modelo elegido, lo que permite seleccionar la más adecuada al problema que tengamos que resolver.

Para obtener un modelo 3D - como por abreviar lo vamos a llamar en adelante- completo por fotogrametría de un monumento es necesario fotografiar, apoyar topográficamente y restituir todas las superficies que son visibles en el mismo. Lógicamente, esto nos obliga a multiplicar exponencialmente el número de $\mathrm{m} 2$ de superficies del edificio sobre las que tenemos que trabajar, ya que es necesario incluir todas las superficies menores, laterales o secundarias que antes eran excluidas en los trabajos habituales de fotogrametría analógica. En estos trabajos, únicamente se dibujaban las superficies que aparecían en los alzados y las secciones principales del edificio y sólo en la orientación del plano representado.

La restitución que se obtiene de la compleja realidad tridimensional es, como sabemos, un conjunto de polilíne- 
as $3 \mathrm{D}$ en el espacio que no forman superficies y, por tanto, son transparentes. Esta circunstancia impide que las superficies que se encuentran en primer plano oculten en las proyecciones las líneas que componen la restitución de las superficies que se encuentran en planos más profundos. Este problema provoca que se produzca la superposición de numerosas líneas en las visualizaciones especialmente complejas del modelo, lo que dificulta su comprensión y su utilidad. Por último, la creación de estos modelos 3D supone la generación de archivos informáticos muy grandes en términos de memoria, lo que dificulta también enormemente su manipulación y operatividad.

\section{DESCOMPOSICIÓN Y ORGANIZACIÓN DEL MODELO 3D}

Para resolver los problemas que provocaban el tamaño de los archivos informáticos y la transparencia de los modelos de líneas, nos dimos cuenta de que era necesario fragmentarlos y descomponerlos en unidades más pequeñas. Esta división del modelo era viable porque estaba todo dibujado en un único sistema de coordenadas, lo que permitía recomponerlo insertando los dibujos unos en otros. Esta característica nos permitía incorporar a la visualización con la que trabajábamos únicamente los archivos que contenían las zonas del edificio que eran visibles. Lógicamente, cuanto mayor era la descomposición del modelo mayores eran las posibilidades de combinación, pero también las dificultades de designación y manipulación de la colección de los archivos resultantes. En los modelos más pequeños — como las iglesias prerrománicas con las que iniciamos nuestro trabajo fotogramétrico- empezamos descomponiéndo el edificio por muros y orientaciones. En el caso de monumentos más grandes como el puente romano de Salamanca o el Acueducto de Segovia, su propia estructura lineal, de un mismo elemento (pila y arco) que se repite indefinidamente, permitía una división muy clara. Sin embargo, una estructura de la complejidad constructiva y espacial y de las dimensiones de un conjunto catedralicio presentaba serias dificultades de fragmentación.

Si miramos en sección uno de estos edificios reconoceremos los elementos constructivos (pilares, arcos, triforio, claristorio, bóvedas y cubiertas) que componen su estructura; si lo hacemos en planta, observaremos un sistema cartesiano adaptado a la geometría real del edificio que está formada por naves y tramos. Este entrecruce de órdenes, el primero arquitectónico y el segundo cartesiano, nos facilitó las claves de cómo tenía que producirse su fragmentación. Dividiremos primero el alzado por funciones constructivas

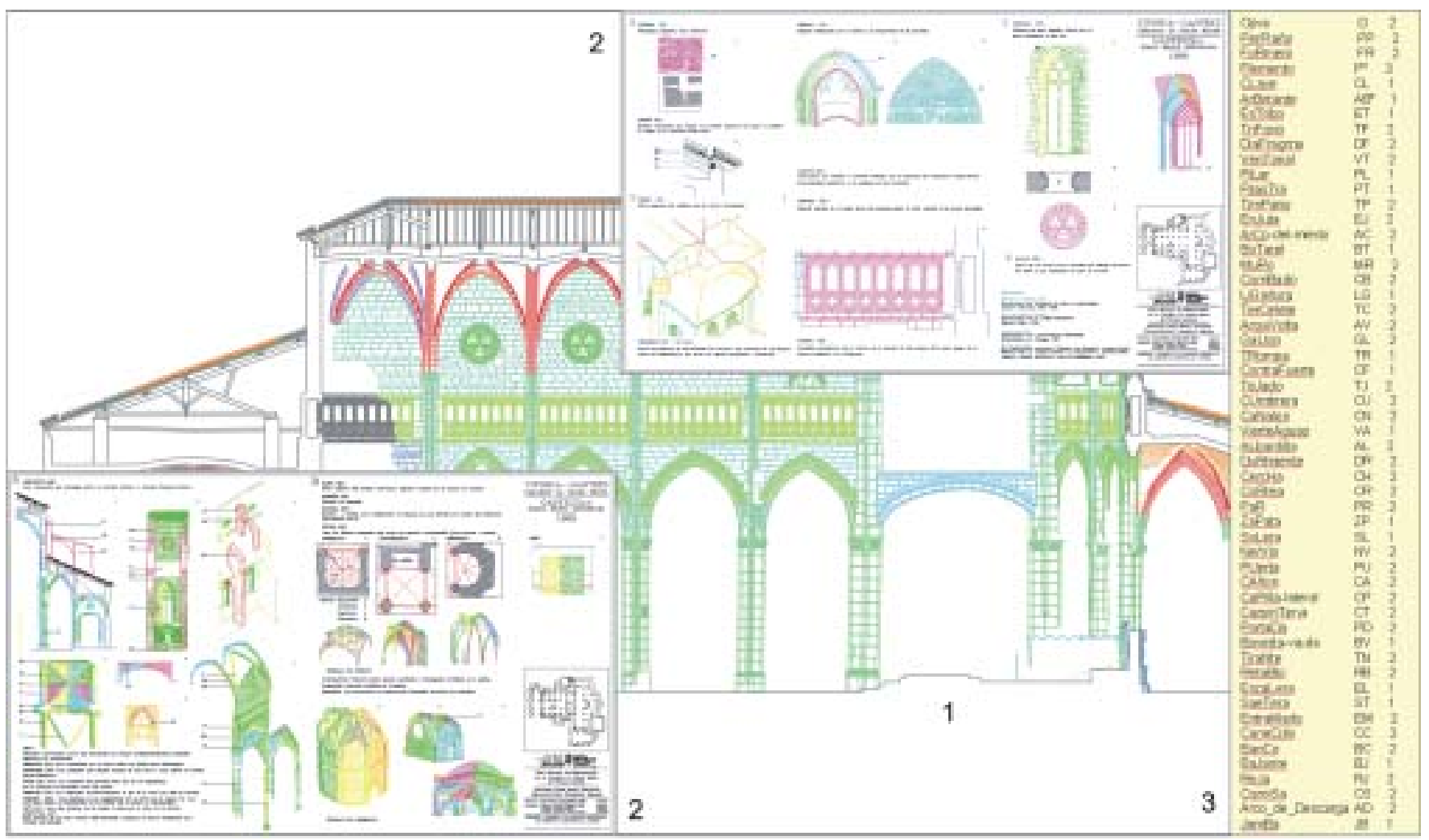

1. Sección longitudinal coloreada por elementos constructivos. 2. Fichas de clasificación tipológica de elementos. 3. Lista de claves de elementos 


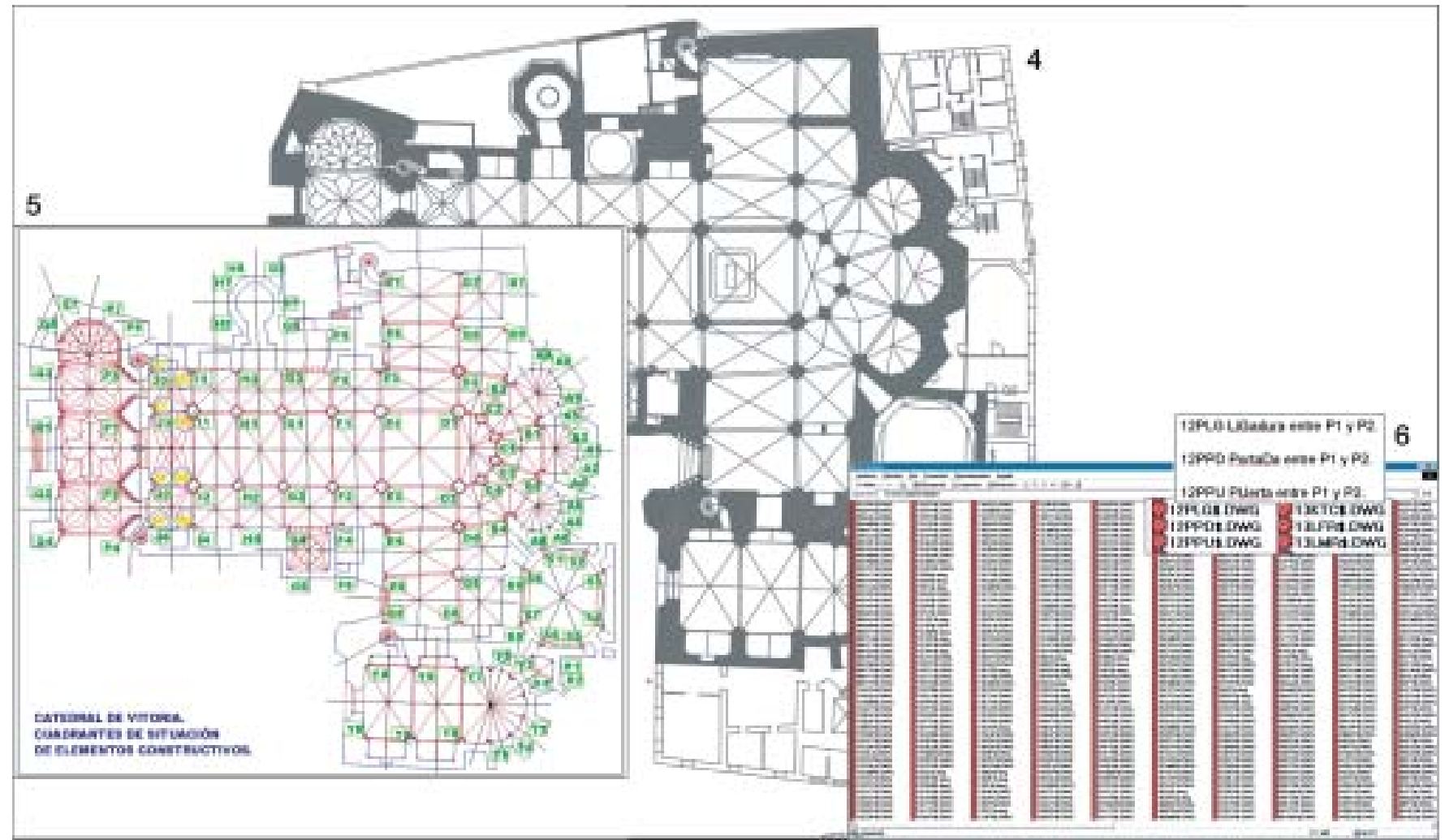

4. Planta del conjunto de la Catedral. 5. Localización de los cuadrantes. 6. Composición de nombres de los elementos por combinación de las divisiones tipológica y topológica

de los miembros: pilares, arcos, muros, bóvedas, arbotantes, estribos, etc. (figs. 1, 2); la planta, en cuadrantes correspondientes a los ejes de la estructura, a los que nombraremos con claves cartesianas de letras y números (en la girola y el presbiterio, la configuración radial nos obligará a un sistema de coordenadas adaptado, anómalo) (figs. 4, 5). La combinación de la clave "tipológica», de función constructiva, con la «topológica», de situación en planta, nos dará claves únicas de designación de cada miembro, a los que hemos llamado «unidades constructivas». La discriminación de qué piedras pertenecen a cada elemento cuando se encuentran imbricados como las pilastras y los muros se hace estableciendo una jerarquía «estructural» de los miembros, en cierto modo ficticia, que establece que la pilastra es más resistente —o conduce más carga - que el muro.

Esta descomposición conduce - en el caso de la Catedral de Vitoria a la que luego nos referiremos- a un modelo formado por más de 1500 archivos de dibujo con nombres distintos que se forman a partir de las claves tipológicas y topológicas del elemento (fig.3, 6). Para facilitar la localización de un archivo dentro de esta colección la hemos dividido primero en dos, según pertenezca la unidad constructiva a la estructura interior o exterior del edificio. La búsqueda de una unidad concreta puede realizarse sim- plemente tecleando el nombre de su archivo (fig. 6). Sin embargo para facilitar esta búsqueda hemos desarrollado un pequeño programa de gestión de los archivos que nos permite reconstruir la clave de designación de la unidad constructiva a partir de la claves tipológicas y topológicas. Para permitir también una búsqueda gráfica de estas unidades hemos desarrollado un modelo simplificado de referencia del monumento que contiene una «línea guía» por cada unidad constructiva. Lo llamamos "plano-guía» porque es como un índice gráfico de lo que contiene el modelo, en el que cada línea tiene el mismo nombre que la unidad a la que representa. Para el manejo del modelo a través del plano-guía hemos programado un menú de órdenes llamado «catedral». Sobre el plano-guía podremos designar con el modo de captura gráfica las líneas guías que representan a los elementos que queremos recuperar. Éstos aparecerán importados en su posición en el espacio, dando lugar a un modelo detallado de una parte del edificio (figs. 10, 11, 12, 13).

Finalmente, las líneas que componen cada uno de estos archivos se estructuran separándolas en capas de dibujo. Esta separación se produce entrecruzando dos niveles de problemas que multiplican el número de estas capas. En primer lugar, realizamos una separación de las líneas por orientaciones geográficas (N, NE, E, SE, S, SW, W, NW); 


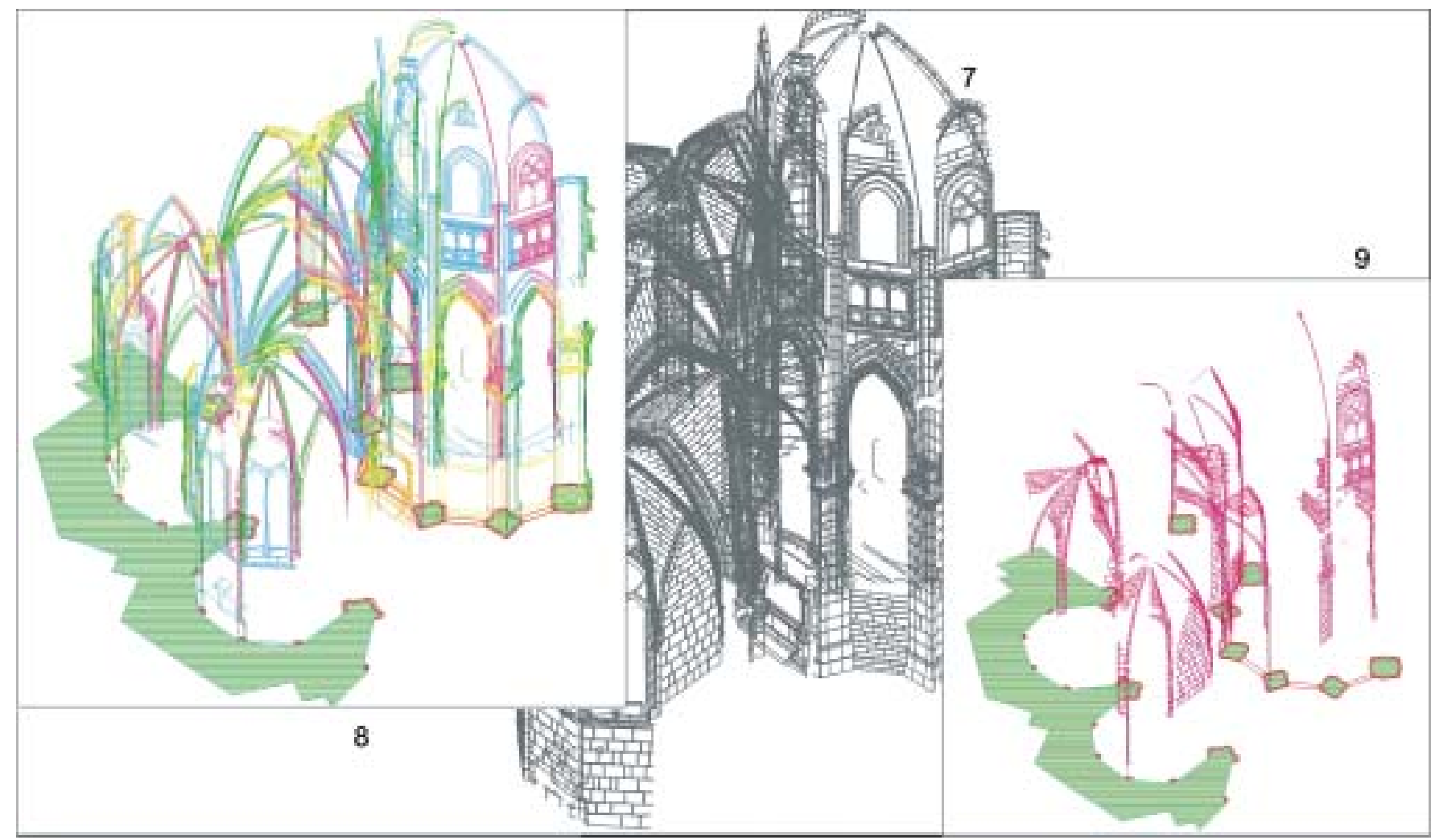

7. Dibujo completo de una parte de la girola de la Catedral. 8. La girola mostrando sólo las aristas arquitectónicas. 9. La girola mostrando sólo las caras de orientación norte

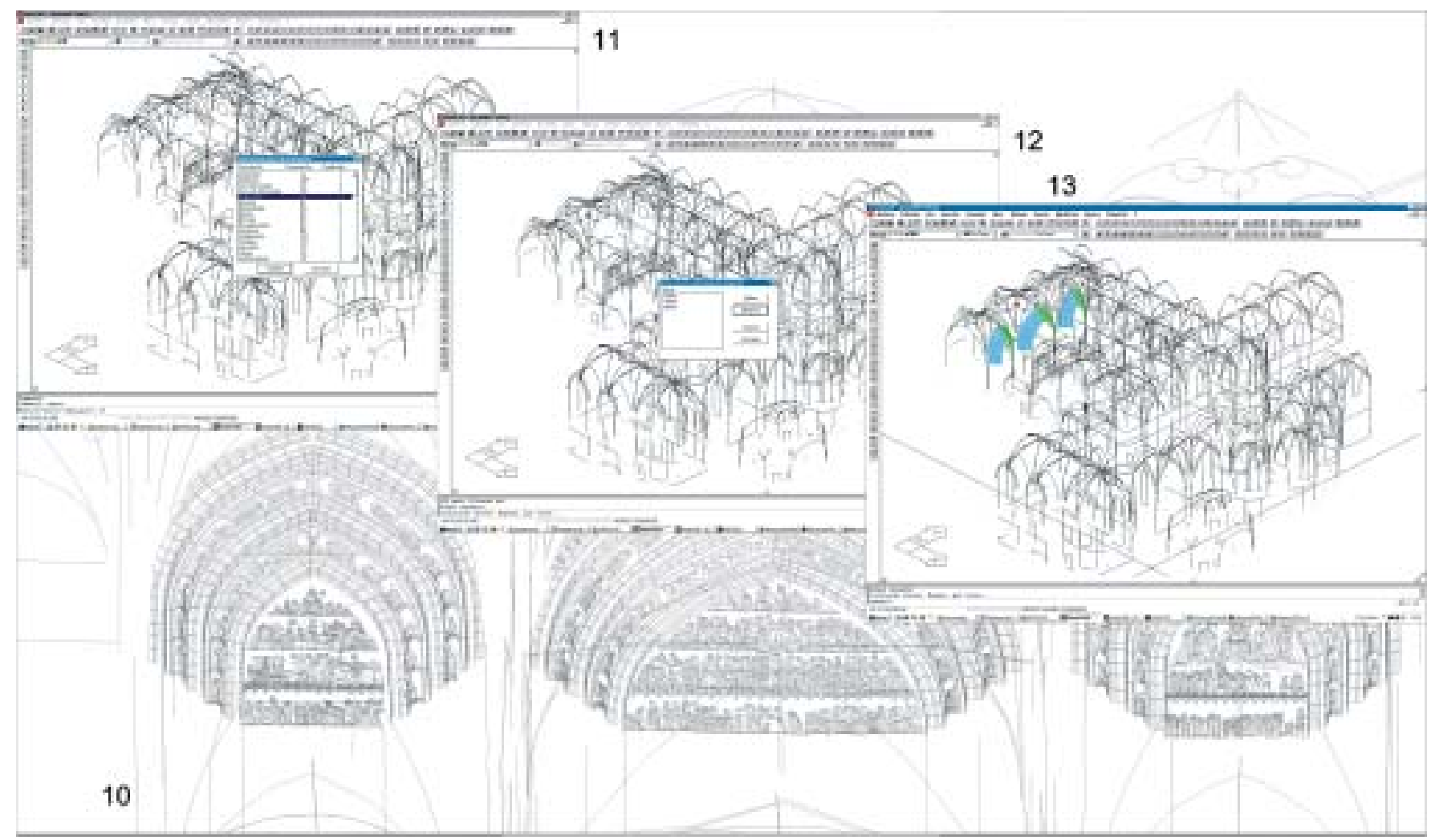

10. Dibujo detallado de las portadas occidentales, importadas en el Plano-guía de la Catedral. 11. Selección de los elementos tipo «arquivolta» en el Plano-guía. 12. Lista de elementos preseleccionados. 13. Importación de los elementos arquivolta seleccionados 
en segundo lugar, la separación se produce por la categoría tipológica de la línea (arista, contorno, fisura, pintura, etc.) y que tiene que ver con el nivel de detalle con el que se dibuja el elemento. Un dibujo, en los que hemos descompuesto el modelo, tendrá tantas capas como orientaciones y categorías tengan las líneas dibujadas; de este modo, podrá existir una capa arista norte o contorno norte, etc. Esta segunda fragmentación del modelo nos permitirá construir dibujos con un nivel de detalle concreto (solo aristas o aristas y contornos, etc.) y en los que únicamente se visualicen las líneas correspondientes a una orientación o varias orientaciones concretas según estemos representando un alzado o una perspectiva (figs. 7, 8, 9).

\section{APLICACIONES Y EXPLOTACIÓN DEL MODELO 3D}

Para explicar las aplicaciones que pueden desarrollarse en el trabajo habitual de restauración arquitectónica con un modelo 3D hemos preferido exponer nuestro trabajo en la Catedral de Santa María de Vitora-Gasteiz. En este trabajo, promovido por la Diputación Foral de Álava y la Fundación Catedral Santa María, en el País Vasco, además de realizar con fotogrametría el modelo 3D del edificio, somos los arquitectos responsables de su restauración, lo que nos ha permitido investigar el desarrollo de un modelo de estas características y explotar personalmente sus aplicaciones. La Catedral de Vitoria es un edificio con una vida agitada, lleno de prótesis y con un estado de deformaciones en su estructura en el límite de lo que razonablemente podemos considerar aceptable. De hecho, el arranque del trabajo de restauración y de toda la investigación realizada se produce por el «miedo» que provocan las deformaciones que sufre el edificio. No entraremos aquí a justificar si esta estructura es estable o no, sino en mostrar como primera aplicación el estudio de esas deformidades. En efecto, el modelo nos ha permitido, no sólo medir la deformación de cada miembro — cosa que se podría hacer arco por arco- (figs. 17, 21) sino, sobre todo, comparar unos arcos con otros dentro del mismo sistema de coordenadas, definiendo las relaciones tridimensionales que se producen entre ellos: los alabeos de los planos intermedios, las pérdidas de curvatura de las ojivas y plementos, etc. (fig. 14). El modelo 3D nos permite insertar, en un nuevo dibujo, los arcos, ojivas y pilastras de una parte o toda la Catedral, o, alternativamente, los muros y plementos que cierran el espacio entre esos elementos lineales (figs. 7, 15, 16). Podemos también comparar medidas reales de un modo que ni siquiera es posible sobre el propio edificio, porque en éste los muros no son transparentes ni traspasables con una cinta métrica.

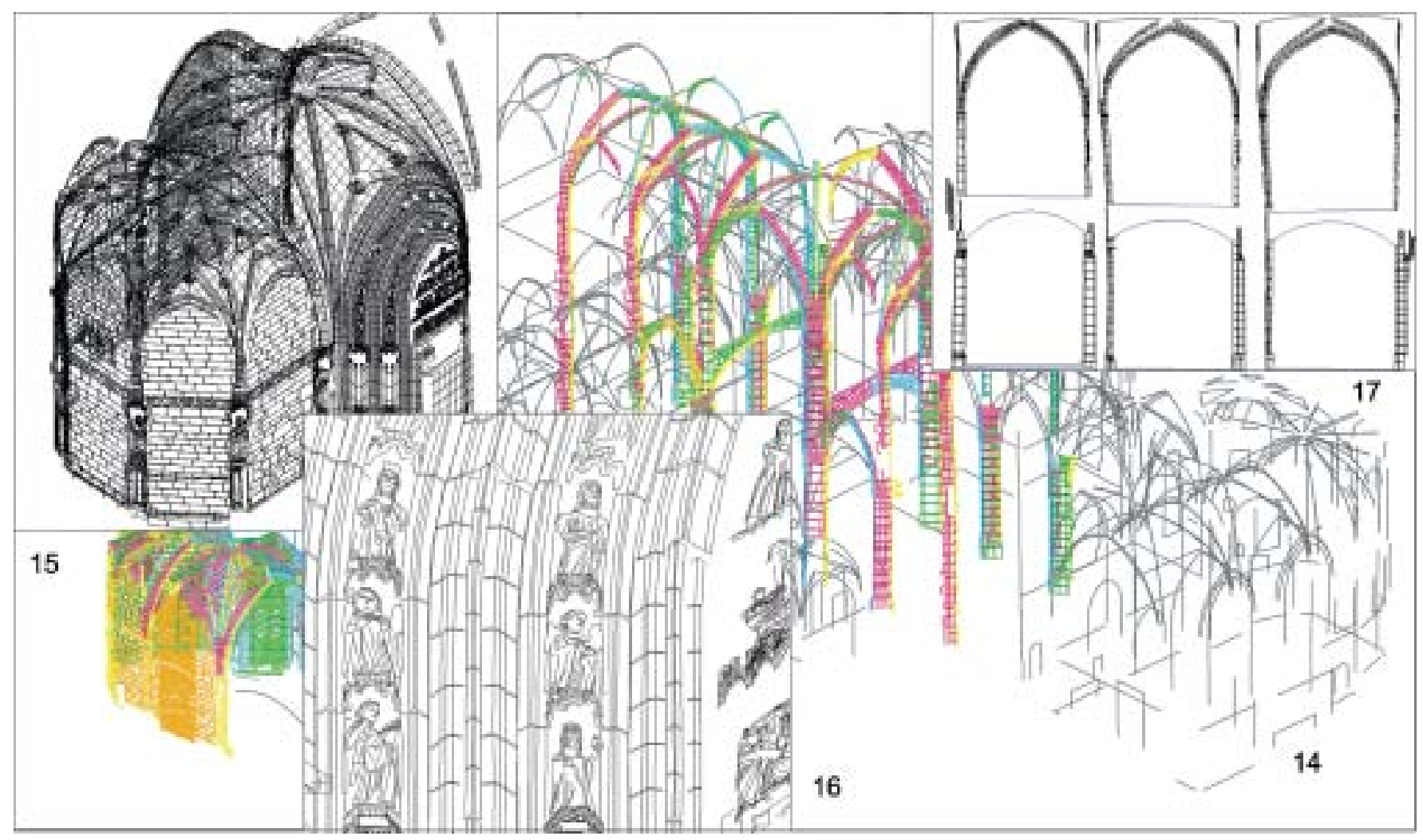

14. Plano-guía con los arcos del transepto y el ábside del pórtico. 15. Detalle del fondo del pórtico. 16. Detalle el dibujo de las arquivoltas del pórtico. 17. Detalle de las deformaciones de los arcos del transepto 


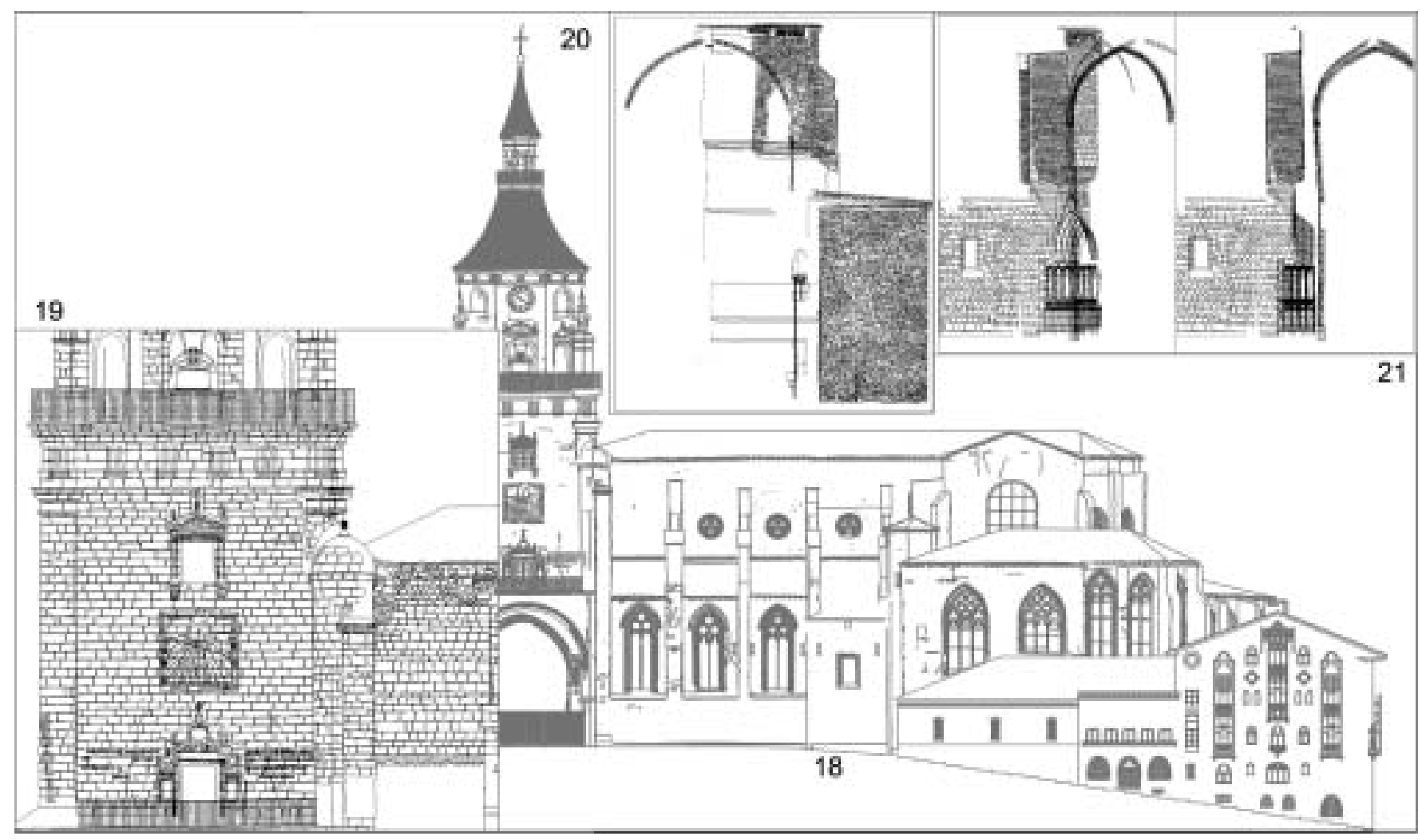

18. Alzado sur de la Catedral. 19.Detalle del alzado de la torre. 20. Proyección de la esquina noreste del transepto en la dirección del arco ojivo y su contrafuerte diagonal. 21. Dos proyecciones del machón de la portada sur para apreciar la deformación sufrida en el tiempo.

Por supuesto, el modelo 3D nos va a permitir agrupar los elementos constructivos que componen una fachada o los volúmenes de una zona concreta del mismo como la torre, la capilla, la nave, etc. o, simplemente, reunir todos los elementos para tener una visión perspectiva del conjunto. Como quiera, que a pesar de aumentar el número de dibujos y la complejidad del modelo 3D no hemos renunciado a la clasificación de menor rango de las líneas con capas y colores correspondientes a las orientaciones y los tipos de línea - juntas, fisuras, aristas, contornos aparentes, etc.-, podemos obtener alzados o secciones sin más que importar los elementos adecuados y activar las capas visibles. Puesto que el modelo es tridimensional y las herramientas de dibujo asistido lo permiten, lo miraremos desde cualquier punto de vista y proyectaremos la visión seleccionada sobre un plano. De este modo, tendremos un producto subsidiario del modelo 3D que son los planos de arquitectura 2D convencionales de alzados, secciones o perspectivas, producto cuya utilidad y facilidad de manejo todos conocemos y que permite desarrollar con más comodidad los planos del proyecto de restauración (figs. 18, 19). Pero con este sistema también podremos obtener secciones "no convencionales", imposibles de obtener si no disponemos de un modelo 3D completo, superponiendo elementos constructivos de dife- rentes planos para estudiar su relación o proyectando elementos diagonales a la estructura general del edifico por un plano paralelo a su desarrollo (fig. 21). Para estudiar el apeo que necesitábamos construir sobre el contrafuerte diagonal de la esquina noreste del transepto y estudiar su relación con el arco ojivo (diagonal) que acomete sobre él, realizamos un alzado-sección por el plano diagonal (fig. 20).

Además de dibujar las líneas de arista de la arquitectura, en este modelo 3D hemos representado también los contornos de cada uno de los materiales visibles en las superficies del mismo. Este trabajo constituye por si solo un análisis constructivo inestimable (figs. 15, 16, 19). Pero además, este modo de representar el edificio permite individualizar e identificar cada material y asignarle un valor específico diferente, mediante tramas o colores. Por ejemplo, de nada nos sirve que reconozcamos 17 litologías diferentes en los muros de la Catedral si no podemos saber cual es la litología específica de cada sillar o mampuesto existente (fig. 25). Tampoco nos sirve de nada que definamos las patologías que sufren estos materiales si no podemos establecer su distribución concreta en los alzados. Esta representación individualizada de los materiales permite también situar con precisión la posición y colocación de los equipos de monitorización o el punto de extracción de determina- 
das muestras para ensayos de laboratorio concretos. Sobre estos alzados constructivos es muy fácil también documentar con precisión el desarrollo de las fisuras existentes y realizar un mapa de las lesiones estructurales (fig. 22). Finalmente, esta representación de los materiales nos ha permitido realizar el análisis arqueológico de la arquitectura de la Catedral asignando a cada material una USM (unidad estratigráfica de muro) concreta $y$, por tanto, un periodo histórico de colocación en el edificio (fig. 23).

Para terminar, diremos que esta herramienta, con todo su poder gráfico, resulta coja sin el concurso de un sistema de almacenamiento de la información obtenida con los estudios del edificio. No nos basta con un estudio cualitativo de los materiales y sus condiciones de conservación, sino que necesitamos su estimación cuantitativa a través de planos temáticos (figs. 23, 25) El Sistema de Información del Monumento MIS (Monument Information System), al que ya nos hemos referido en el punto segundo, establece una relación informática entre los valores y características obtenidas en los estudios (base de datos) y unos valores topológicos definidos por el contorno gráfico con el que hemos representado cada uno de los materiales del edificio (modelo 3D). Mediante esta relación se establece un sistema interactivo de consultas de doble vía entre los datos y valores obtenidos y su relación topológica mensurable en el modelo 3D (figs. 23, 24, 25). Este Sistema de Información de los Monumentos MIS (Monument Information System) constituye - por su contenido y configuración - un sistema completo de documentación de un monumento. Pero además, por su condición de sistema abierto, permite su manipulación y utilización en los procesos de proyecto y su revisión y actualización durante el desarrollo de la obra, lo que lo convierte en una herramienta de valor inestimable en la disciplina de la restauración de monumentos.

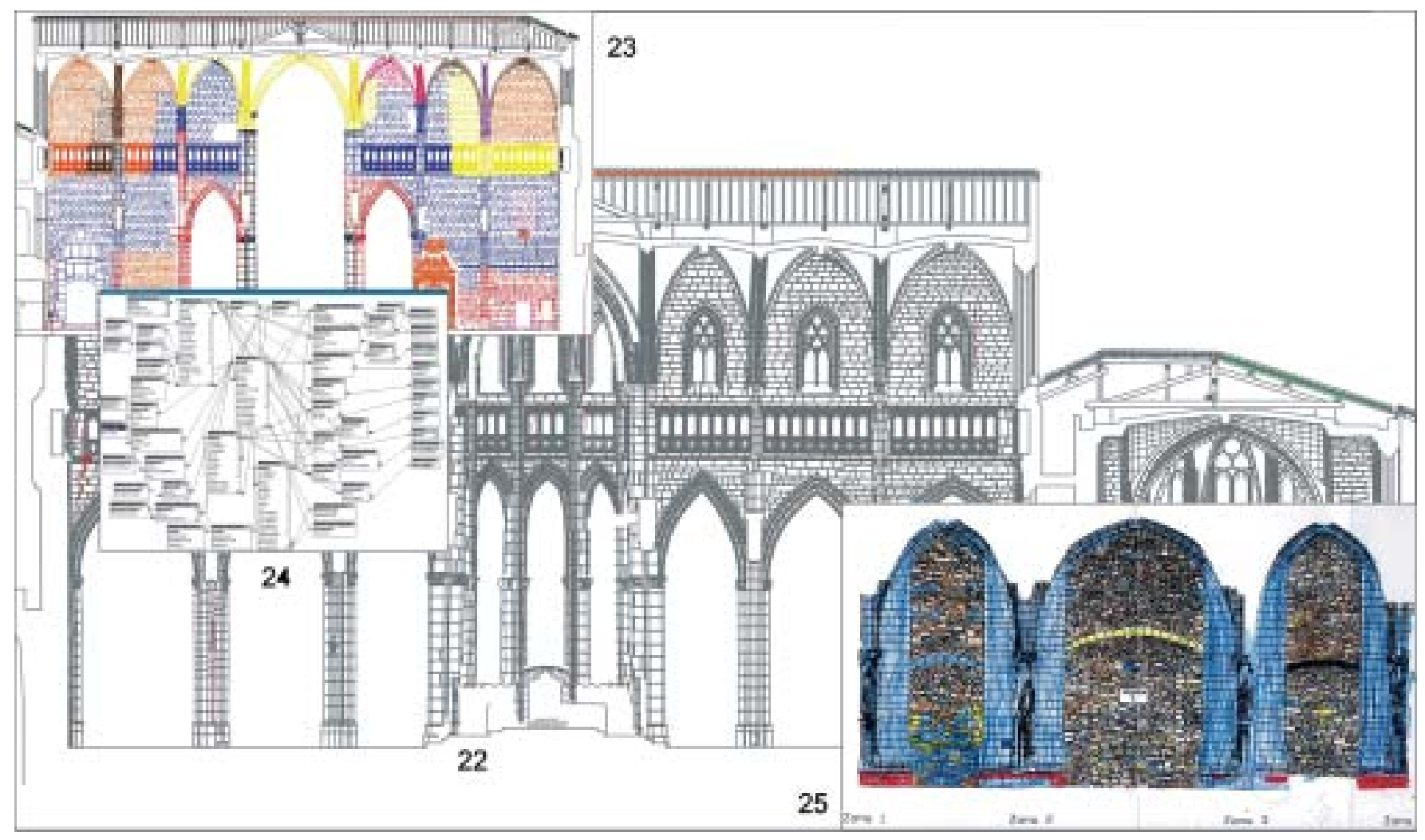

22. Sección del transepto con análisis de las lesiones estructurales. 23. Sección del transepto coloreada por etapas constructivas históricas. 24. Estructura interna de la base de datos histórica, con cuatro tablas principales y 46 secundarias. 25. Tipos de piedra usados en la construcción del pórtico occidental 


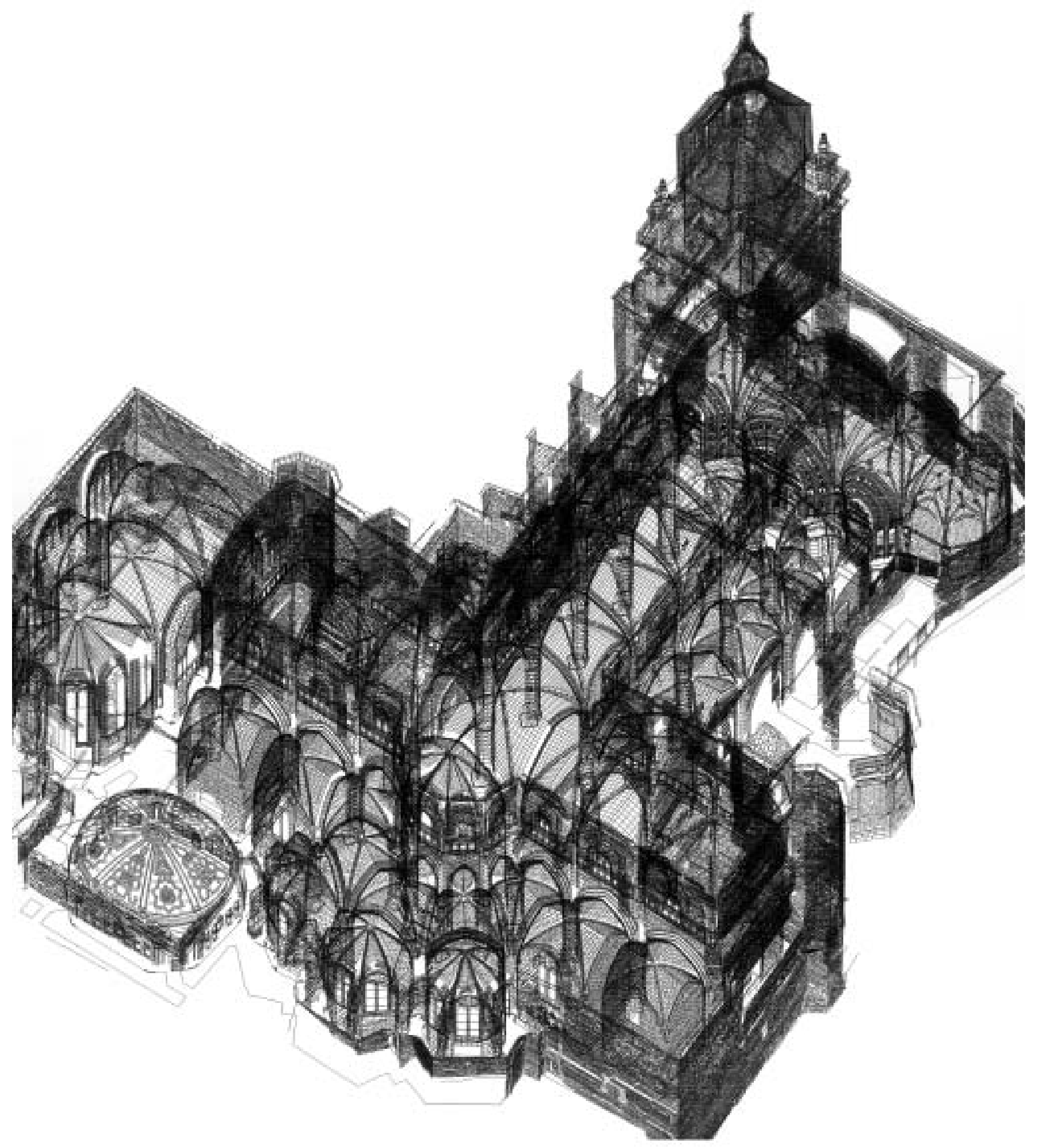

